CLINICAL STUDY

\title{
Association study of AMP-activated protein kinase subunit genes in polycystic ovary syndrome
}

\author{
Kari Sproul $^{1,2}$, Michelle R Jones ${ }^{3}$, Ricardo Azziz ${ }^{1,2,4}$ and Mark O Goodarzi ${ }^{1,3,4,5}$ \\ ${ }^{1}$ Department of Obstetrics and Gynecology, Cedars-Sinai Medical Center, Los Angeles, California 90048, USA, ${ }^{2}$ Department of Obstetrics and Gynecology, \\ the David Geffen School of Medicine at UCLA, Los Angeles, California 90095, USA, ${ }^{3}$ Division of Endocrinology, Diabetes and Metabolism, Department of \\ Medicine, Cedars-Sinai Medical Center, 8700 Beverly Boulevard, Room B-131, Los Angeles, California 90048, USA, ${ }^{4}$ Department of Medicine, the David \\ Geffen School of Medicine at UCLA, Los Angeles, California 90095, USA and ${ }^{5}$ Medical Genetics Institute, Cedars-Sinai Medical Center, Los Angeles, \\ California 90048, USA \\ (Correspondence should be addressed to M O Goodarzi at Division of Endocrinology, Diabetes and Metabolism, Department of Medicine, Cedars-Sinai \\ Medical Center; Email: mark.goodarzi@cshs.org)
}

\begin{abstract}
Objective: To examine the genes for AMP-activated protein kinase (AMPK) subunits $\alpha 2$ (PRKAA2) and $\gamma 3$ (PRKAG3) as candidates for polycystic ovary syndrome (PCOS) and its component traits.

Design and methods: A total of 287 white PCOS women were recruited from the reproductive endocrinology clinic at the University of Alabama at Birmingham and 187 white control subjects were recruited from the surrounding community. Seven PRKAA2 single nucleotide polymorphisms (SNPs) and four PRKAG3 SNPs were genotyped in PCOS cases and controls. Genotyping and association analysis were performed at Cedars-Sinai Medical Center.

Results: Nominal associations of PRKAA2 variants with insulin-related traits and the PRKAG3 Pro71Ala variant with PCOS were not statistically significant after multiple testing correction. Among PCOS patients, there were no associations between variants in AMPK subunit genes and androgenic or reproductive traits.

Conclusions: Variants in genes for AMPK $\alpha 2$ and AMPK $\gamma 3$ were not associated with PCOS or its component traits. Our evidence does not demonstrate that AMPK is a major genetic risk factor for PCOS.
\end{abstract}

European Journal of Endocrinology $161405-409$

\section{Introduction}

Polycystic ovary syndrome (PCOS) is a common complex genetic disorder, affecting $\sim 6-8 \%$ of reproductive age women $(1,2)$. PCOS is characterized by hyperandrogenism, ovulatory dysfunction, polycystic ovarian morphology, and frequently insulin resistance (IR).

AMP-activated protein kinase (AMPK) is a cellular energy sensor, acting to maintain ATP levels in the cell by sensing the AMP/ATP ratio and then phosphorylating metabolic enzymes and regulating gene expression with the net result of activating processes that generate ATP and inhibiting processes that consume ATP. AMPK influences food intake, fatty acid and glucose uptake in skeletal and cardiac muscle, insulin secretion, and hepatic fatty acid synthesis and gluconeogenesis (3). Consequently, AMPK subunit genes have been examined as candidates for type 2 diabetes susceptibility, with conflicting results $(4,5)$.

As PCOS is a metabolic disorder associated with an increased risk of diabetes, AMPK may play a role in this association. Metformin, commonly prescribed for PCOS patients, activates AMPK activity in skeletal muscle (6). In addition, AMPK activation appears to be decreased in
PCOS ovarian theca cells, suggesting that metformin stimulation of AMPK in the ovary itself may promote ovulation (7). We therefore examined the genes for AMPK $\alpha 2$ (PRKAA2) and AMPK 33 (PRKAG3) as candidate genes for PCOS and its component traits.

\section{Subjects and methods}

\section{Subjects and phenotyping}

A total of 287 consecutive unrelated white patients with PCOS, aged 13-47 years, and 187 healthy unrelated white control women, aged 14-60, were recruited from the Birmingham, AL, USA area. PCOS cases were recruited from the reproductive endocrine practice of one of the investigators (RA) at the University of Alabama at Birmingham (UAB). All subjects were non-Hispanic Caucasians. Participation in research studies was offered to patients meeting inclusion criteria (premenopausal, not pregnant, on no hormonal therapy for at least 3 months, and meeting diagnostic criteria for PCOS). PCOS was diagnosed following the 1990 NIH consensus criteria (8). 
Controls were healthy women with a history of regular menstrual cycles and no family history of hirsutism. These women had no evidence of hirsutism, acne or alopecia, or endocrine dysfunction and had not taken hormonal therapy (including oral contraceptives) for at least 3 months prior to testing. Controls were recruited by word of mouth and advertisements in the Birmingham, Alabama area, through a call for 'healthy women' without detailing further the nature of the studies.

Subjects underwent a physical examination and serum androgen measurement, as per a previously described protocol (1). Fasting glucose and insulin were also obtained in a subset of the cohort $(\sim 70 \%)$. This subset did not differ demographically or hormonally from the study subjects overall. The computer-based homeostasis model assessment (HOMA, www.dtu.ox.ac. uk/homa) utilizes fasting glucose and insulin to calculate indices of IR (HOMA-IR) and insulin secretion (HOMA-\%B) (9). For the insulin-related traits only, six PCOS subjects with diabetes were excluded because the hyperglycemia of diabetes may induce secondary changes in insulin-related traits that reduce their utility for genetic analysis. In addition, in PCOS cases only, we evaluated the following reproductive traits: severe oligomenorrhea (defined as $>3$ months between menses), infertility (yes/no), and parity (yes/no). Clinical characteristics of the subjects are given in supplementary Table 1, which can be viewed online at http://www.eje-online.org/supplemental/.

All subjects gave written informed consent, and the study was performed according to the guidelines of the Institutional Review Boards of UAB and CedarsSinai Medical Center, Los Angeles, CA, USA.

\section{Genetic analysis}

We selected six PRKAA2 single nucleotide polymorphisms (SNPs; rs11206887, rs2143749, rs2746349, rs12749128, rs857155, and rs3738568) using genotype data of the Caucasian population of the HapMap database (release 24, phase II) (10). These SNPs capture 28 out of $35(80 \%)$ of the Caucasian HapMap SNP alleles at $r^{2}>0.8$. An additional PRKAA2 SNP (rs2051040) was selected because it was associated with type 2 diabetes in a Japanese cohort (4). Four validated PRKAG3 SNPs (rs6436094, rs16859382, rs692243, and rs650898) were genotyped, selected based on frequency $>5 \%$ because only two SNPs in HapMap (rs6436094, rs692243) are polymorphic in Caucasians. SNP rs692243 is a nonsynonymous variant (proline to alanine at codon 71). The eleven SNPs were genotyped in the 474 subjects using the 5'-exonuclease assay (TaqMan MGB, Applied Biosystems, Foster City, CA, USA). Supplementary Table 2, which can be viewed online at http://www. eje-online.org/supplemental/ lists PCR primers and TaqMan MGB probes.

\section{Statistical analysis}

Unpaired $t$-tests and $\chi^{2}$ tests were used to compare clinical characteristics between women with and without PCOS; quantitative trait values were log- or square root-transformed as appropriate to reduce nonnormality.

Association with the presence/absence of PCOS and qualitative traits was evaluated using logistic regression, adjusting for age and body mass index (BMI) by including both as independent variables. Association with quantitative traits was evaluated using analysis of covariance, again adjusting for age and BMI. Recognizing that insulin secretion responds to ambient IR, HOMA-\%B associations were also calculated with HOMA-IR as a covariate in addition to age and BMI. Quantitative trait associations were conducted in the PCOS and control groups separately. To handle multiple testing, significance was taken as $P<0.006$, considering that we analyzed two genes against four families of traits (PCOS diagnosis, androgens, metabolic traits and reproductive endpoints), yielding a Bonferroni correction factor of 8 (i.e. eight independent comparisons). Association analyses excluding 26 post-menopausal subjects from the control group yielded the same results as analyses conducted in the entire cohort; therefore, the latter results are reported.

\section{Results}

\section{PRKAA2}

We genotyped seven SNPs spanning the PRKAA2 gene (Fig. 1A). SNP frequencies are shown in Table 1. All markers were in Hardy-Weinberg equilibrium. Linkage disequilibrium among these markers $\left(D^{\prime}\right)$ ranged from 0.45 to 1.0 (average pairwise $D^{\prime}$ of 0.90 ). The $r^{2}$ values ranged from 0 to 0.98 (average 0.23 ).

Among the women with PCOS, carriers of the minor alleles of SNPs rs2143749, rs2746349, or rs857155 had higher fasting insulin levels than noncarriers $(P=0.044,0.021$, and 0.027 respectively). Carriers of the minor allele of SNP rs2143749 also had increased HOMA-\%B $(P=0.030)$, which was not significant when HOMA-IR was also included as a covariate $(P=0.26)$. Lastly, PCOS women who were carriers of the minor allele of SNP rs2746349 had increased HOMA-IR compared with noncarriers $(P=0.039)$. Trait values for PCOS women are shown in the electronic supplemental Table 3, which can be viewed online at http:// www.eje-online.org/supplemental/. None of these PRKAA2 associations were significant after considering multiple testing. We did not find significant associations between PRKAA2 variants and PCOS itself, BMI, androgens, or reproductive traits. No significant associations were observed with quantitative traits in control subjects. 


\section{A PRKAA2}

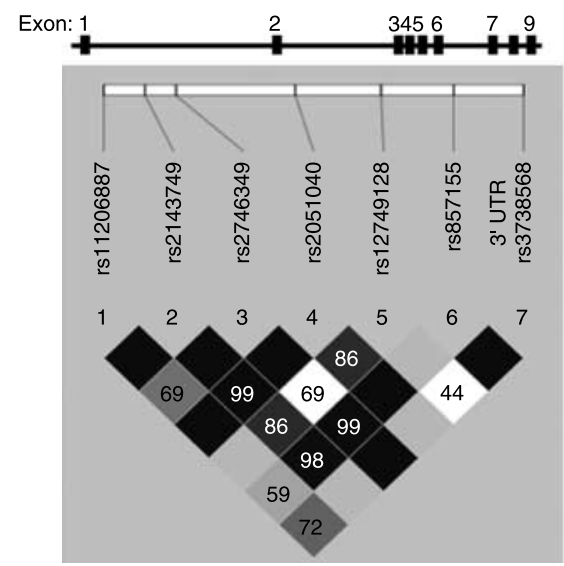

B PRKAG3

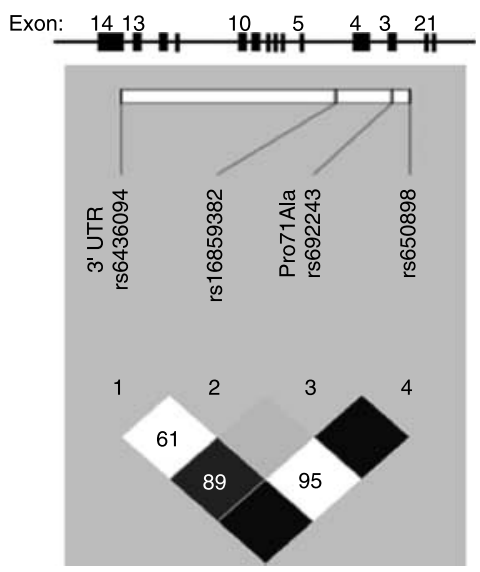

Figure 1 Gene structure and linkage disequilibrium plots for PRKAA2 (A) and PRKAG3 (B). Gene structure is shown at the top. PRKAA2 has nine exons and is located on chromosome 1 (1p31). PRKAG3 has 14 exons and is located on the reverse (-) strand of chromosome 2 (2q35).

The locations of the genotyped SNPS relative to the exons are indicated. The linkage disequilibrium plots at the bottom display $D^{\prime}$ values (\%) for each pair of SNPs in the box at the intersection of the diagonals from each SNP. The dark solid blocks indicate $D^{\prime}=1(100 \%)$ for the corresponding pair of variants. The lighter solid boxes also indicate $D^{\prime}=1$, but with a low confidence score. $3^{\prime}$ UTR indicates SNP located in the $3^{\prime}$ UTR.

\section{PRKAG3}

We genotyped four SNPs spanning the PRKAG3 gene (Fig. 1B). The genotype frequencies (Table 1) for all markers were in Hardy-Weinberg equilibrium. Linkage disequilibrium among the four markers $\left(D^{\prime}\right)$ ranged from 0.61 to 1.0 (average 0.91 ); $r^{2}$ values ranged from 0.001 to 0.55 (average 0.10 ).

Carriers of the minor allele of SNP rs692243 (Pro71Ala) had an increased likelihood of PCOS, with an age- and BMI-adjusted odds ratio of 1.82 (95\% confidence interval, CI, 1.07-3.10, $P=0.028$ ), which did not meet the multiple testing-corrected $P$ value cutoff of 0.006. We found no associations in PCOS or control women between the PRKAG3 variants and any of the quantitative traits, including BMI.

\section{Discussion}

This is the first study evaluating association of variants in the PRKAA2 and PRKAG3 genes with PCOS. Nominal associations of PRKAA2 variants with insulin-related traits and of the PRKAG3 Pro71Ala variant with PCOS were not statistically significant after correcting for multiple testing.

AMPK is a heterotrimeric enzyme composed of a catalytic alpha subunit $(\alpha)$ and two noncatalytic regulatory $(\beta$ and $\gamma)$ subunits. Seven genes code for the possible components of AMPK $(\alpha 1, \alpha 2, \beta 1, \beta 2, \gamma 1$, $\gamma 2$, and $\gamma 3$ ). We considered PRKAA2 and PRKAG3, which code for the $\alpha 2$ and $\gamma 3$ subunits, as the highest priority candidate genes, given prior physiologic evidence implicating their proteins in metformin action (6) and insulin sensitivity $(11,12)$.

No other human genetic association studies of AMPK in PCOS have been published. A few human genetic studies examined AMPK in nonPCOS cohorts with conflicting results. Some demonstrated associations between PRKAA2 variants and type 2 diabetes, HOMA-IR, and cholesterol fractions $(4,13,14)$. One study found no association of variation in PRKAA2 with type 2 diabetes, fasting glucose, IR, insulin secretion, or body fat distribution (5). Our PRKAA2 results are consistent with the latter study.

Table 1 Frequency and position information on PRKAA2 and PRKAG3 single nucleotide polymorphisms (SNPs).

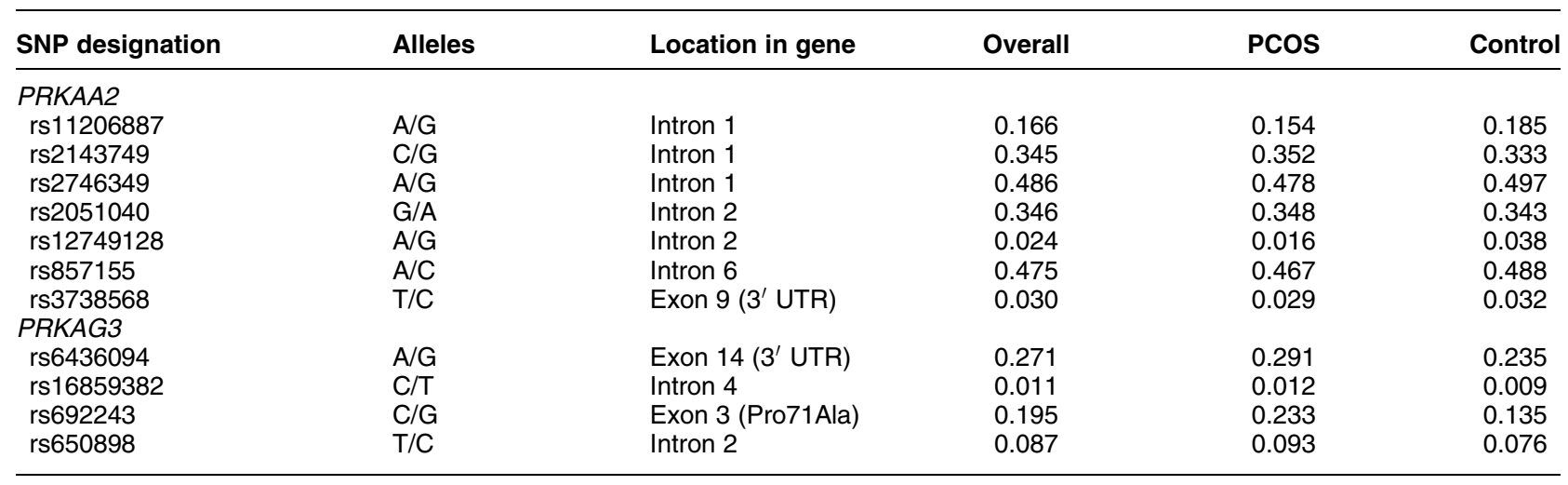


PRKAG3 variants were not associated with insulin sensitivity or secretion in prior studies (15), similar to our current results in PCOS women. Previously, the minor allele of SNP rs692243, which is a nonsynonymous variant (proline-to-alanine at codon 71), was shown to be significantly associated with higher serum LDL-cholesterol and apolipoprotein B-100 levels, supporting the functional significance of this SNP (15). We do not have cholesterol levels for our patients, but we did observe that this same SNP, rs692243, was marginally associated with PCOS itself.

Studies conducted primarily in animals documented AMPK expression in the ovary, suggesting a role for this fuel sensor in fertility (16). Recent work has found expression of AMPK in human ovaries with AMPK activation decreased in PCOS thecal cells, and that metformin treatment of these cells increased AMPK activity (7). Thus, activation of AMPK in the ovary itself could be a reason why patients with PCOS who are treated with metformin experience amelioration of ovulatory dysfunction. However, variants in the AMPK subunit genes we analyzed were not associated with ovulatory dysfunction in PCOS patients as assessed by severe oligomenorrhea, infertility, and/or parity. As reproductive traits are interrelated with metabolic traits in PCOS, the association of reproductive traits with AMPK SNPs was predictably lacking in the absence of any association of metabolic traits with these SNPs.

The value of our study is that it reports on genes that have not yet been evaluated in PCOS patients, in a homogeneous and well-characterized case/control cohort. We have performed a number of genetic association studies in this cohort from Alabama. While this may seem duplicative, it is critical to completely assess a well-defined population, minimizing the variability brought on by analyzing different genes in different populations. Thus, it is common in genetic epidemiology to use a single cohort for ongoing investigation and to report each investigation as a separate experiment, when that is how the work was performed. For example, many genetic association publications have resulted from the Insulin Resistance Atherosclerosis Family Study and the Multi-ethnic Study of Atherosclerosis.

A limitation of the current study is that IR and insulin secretion were measured by HOMA indices, which may be insufficiently reflective of these traits to allow detection of association with genetic variants, particularly in the setting of PCOS (17). This may have hindered our ability to discover robust associations of AMPK subunit genetic variants with insulin-related traits. Analysis in a cohort characterized by physiologic studies (e.g. euglycemic clamp) would be necessary to definitively rule out an association of PRKAA2 or PRKAG3 variants with IR.

In conclusion, variants in the genes for AMPK $\alpha 2$ and AMPK $\gamma 3$ were not associated with PCOS susceptibility or the component metabolic, reproductive, or androgenic traits in PCOS patients, suggesting that PRKAA2 and PRKAG3 are not major genetic risk factors for PCOS. Whether genetic variants in other AMPK subunit genes play a role in PCOS susceptibility remains to be determined.

\section{Declaration of interest}

There are no conflicts of interest related to the content of this work.

\section{Funding}

This study was supported in part by NIH grants R01-HD29364 and K24-HD01346 (to R Azziz), R01-DK79888 (to M O Goodarzi), and M01-RR00425 (General Clinical Research Center Grant from the NCRR), and an endowment from the Helping Hand of Los Angeles, Inc.

\section{References}

1 Azziz R, Woods KS, Reyna R, Key TJ, Knochenhauer ES \& Yildiz BO. The prevalence and features of the polycystic ovary syndrome in an unselected population. Journal of Clinical Endocrinology and Metabolism 200489 2745-2749.

2 Diamanti-Kandarakis E, Kouli CR, Bergiele AT, Filandra FA, Tsianateli TC, Spina GG, Zapanti ED \& Bartzis MI. A survey of the polycystic ovary syndrome in the Greek island of Lesbos: hormonal and metabolic profile. Journal of Clinical Endocrinology and Metabolism $1999 \mathbf{8 4} 4006-4011$.

3 Kahn BB, Alquier T, Carling D \& Hardie DG. AMP-activated protein kinase: ancient energy gauge provides clues to modern understanding of metabolism. Cell Metabolism 2005 1 15-25.

4 Horikoshi M, Hara K, Ohashi J, Miyake K, Tokunaga K, Ito C, Kasuga M, Nagai R \& Kadowaki T. A polymorphism in the AMPKalpha2 subunit gene is associated with insulin resistance and type 2 diabetes in the Japanese population. Diabetes 200655 919-923.

5 Sun MW, Lee JY, de Bakker PI, Burtt NP, Almgren P, Rastam L, Tuomi T, Gaudet D, Daly MJ, Hirschhorn JN, Altshuler D, Groop L \& Florez JC. Haplotype structures and large-scale association testing of the $5^{\prime}$ AMP-activated protein kinase genes PRKAA2, PRKAB1, and PRKAB2 with type 2 diabetes. Diabetes 200655 $849-855$.

6 Musi N, Hirshman MF, Nygren J, Svanfeldt M, Bavenholm P, Rooyackers O, Zhou G, Williamson JM, Ljunqvist O, Efendic S, Moller DE, Thorell A \& Goodyear LJ. Metformin increases AMPactivated protein kinase activity in skeletal muscle of subjects with type 2 diabetes. Diabetes $2002512074-2081$.

7 McAllister J. Stress signaling in the PCOS ovary. In Proceedings of the 4th Special Scientific Meeting of the Androgen Excess and PCOS Society, p 9. San Francisco, CA, 2008.

8 Zawadzki JK \& Dunaif A. Diagnostic criteria for polycystic ovary syndrome: towards a rational approach. In Polycystic Ovary Syndrome, pp 377-384. Eds A Dunaif, JR Givens, F Haseltine \& GR Merriam, Boston: Blackwell Scientific, 1992.

9 Levy JC, Matthews DR \& Hermans MP. Correct homeostasis model assessment (HOMA) evaluation uses the computer program. Diabetes Care 199821 2191-2192.

10 The International HapMap Consortium. The International HapMap Project. Nature 2003426 789-796.

11 Barnes BR, Marklund S, Steiler TL, Walter M, Hjalm G, Amarger V, Mahlapuu M, Leng Y, Johansson C, Galuska D, Lindgren K, Abrink M, Stapleton D, Zierath JR \& Andersson L. The 5'-AMPactivated protein kinase gamma 3 isoform has a key role in carbohydrate and lipid metabolism in glycolytic skeletal muscle. Journal of Biological Chemistry $200427938441-38447$.

12 Viollet B, Andreelli F, Jorgensen SB, Perrin C, Geloen A, Flamez D, Mu J, Lenzner C, Baud O, Bennoun M, Gomas E, Nicolas G, 
Wojtaszewski JF, Kahn A, Carling D, Schuit FC, Birnbaum MJ, Richter EA, Burcelin R \& Vaulont S. The AMP-activated protein kinase alpha2 catalytic subunit controls whole-body insulin sensitivity. Journal of Clinical Investigation 2003 111 91-98.

13 Keshavarz P, Inoue H, Nakamura N, Yoshikawa T, Tanahashi T \& Itakura M. Single nucleotide polymorphisms in genes encoding LKB1 (STK11), TORC2 (CRTC2) and AMPK alpha2-subunit (PRKAA2) and risk of type 2 diabetes. Molecular Genetics and Metabolism 200893 200-209.

14 Spencer-Jones NJ, Ge D, Snieder H, Perks U, Swaminathan R, Spector TD, Carter ND \& O'Dell SD. AMP-kinase alpha2 subunit gene PRKAA2 variants are associated with total cholesterol, lowdensity lipoprotein-cholesterol and high-density lipoproteincholesterol in normal women. Journal of Medical Genetics 2006 43 936-942.

15 Weyrich P, Machicao F, Staiger H, Simon P, Thamer C, Machann J. Schick F, Guirguis A, Fritsche A, Stefan N \& Haring HU. Role of
AMP-activated protein kinase gamma 3 genetic variability in glucose and lipid metabolism in non-diabetic whites. Diabetologia $2007502097-2106$.

16 Dupont J, Chabrolle C, Rame C, Tosca L \& Coyral-Castel S. Role of the peroxisome proliferator-activated receptors, adenosine monophosphate-activated kinase, and adiponectin in the ovary. PPAR Research 20082008 1-9.

17 Diamanti-Kandarakis E, Kouli C, Alexandraki K \& Spina G. Failure of mathematical indices to accurately assess insulin resistance in lean, overweight, or obese women with polycystic ovary syndrome. Journal of Clinical Endocrinology and Metabolism 2004 89 1273-1276.

Received 17 June 2009

Accepted 29 June 2009 